\title{
Cavitational damage of titanium in molten lead
}

\author{
A M ABDEL FATTAH and M A SIDKEY \\ National Institute of Standards, Cairo, Egypt
}

MS received 9 August 1982

\begin{abstract}
Cavitation erosion of pure titanium metal in molten lead was measured at temperatures varying between 350 and $800^{\circ} \mathrm{C}$. Magnetostrictive transducer was used to generate ultrasonic waves at frequency $200 \mathrm{kHz}$ and driving amplitude of $4 \mu \mathrm{m}$. The results showed an increase in the weight loss of titanium with increase in both temperature and cavitation noise level. The starting temperature for dissolving titanium in lead changed from $725^{\circ}$ at normal conditions, to $600^{\circ} \mathrm{C}$ when ultrasonic field was applied.
\end{abstract}

Keywords. Cavitational damage; titanium; cavitation erosion.

\section{Introduction}

It is generally known that solid surfaces immersed in a cavitating liquid suffer from severe erosion. High intensity sound fields in liquids generate cavitation, which is the process of expansion and subsequent violent collapse of minute gas bubbles present in the liquid. The mechanical stress arising from the motion of the cavitating bubbles, and the shock waves associated with their collapse bring about permanent damage to the surface of the solid (erosion).

Analysis of cavitation noise has shown that spectral components provide valuable information on the evolution of cavitation process (Esche 1952). The relation between cavitation erosion of aluminium and cavitation noise level in water was reported by Bebchuk et al (1958) as a linear relation.

The magnitude of cavitation erosion depends on the amount of generating cavitation bubbling and the rate of collapse (Bebchuk 1957), which in turn determines the power of the shock wave obtained as a result of collapse. Rosenberg (1960) and Ibisi (1967), reported a steady increase in the weight loss with rise in temperature to a peak at $50^{\circ} \mathrm{C}$ followed by a decrease in weight loss with further increase in temperature. The erosion rate is also affected by many factors such as; acoustic power, frequency, exposure time and physicochemical properties of both cavitating liquid and solid surface immersed in the liquid.

Although much work has been done on cavitation erosion at moderate temperatures in liquids, few results were reported at high temperature in molten metals. In this paper, an attempt is made to find out the relation between cavitation erosion of titanium in molten lead and the temperature of the melt. The connection between cavitation erosion and both cavitation noise spectrum and cavitation threshold pressure is also discussed. 


\section{Experimental}

Ultrasonic cavitation was excited in molten lead using a magnetostrictive nickel alloy transducer of frequency $20 \mathrm{kHz}$ manufactured by $\mathrm{Dr}$ Lehfeldt and Co. The transducer was fitted with a half wavelength velocity transformer joined to a half wavelength coupling titanium rod which acts as a radiator. This titanium rod was dipped directly $1 \mathrm{~cm}$ below the surface of molten lead.

The melt was kept in a graphite crucible which can accommodate about 1600 $\mathrm{cm}^{3}$ of molten lead. The temperature of the melt was measured with a $\mathrm{Ni}, \mathrm{Ni}-\mathrm{Cr}$ calibrated thermocouple inserted into the melt beside the vibrating rod until the desired temperature was reached and stabilized and was then taken away.

The noise produced by cavitation in the melt was detected with a small piezoelectric microphone, attached to a waveguide probe, used as a receiver dipped in the melt. The signal from the microphone was fed to the Tektronix plug of a spectrum analyser fitted with a sweeping local oscillator. The spectrograms were recorded on a Hewlett-Packard X-Y recorder whose tap-derive mechanism was mechanically linked to the output of the analyser.

Two series of experimental measurements were carried out. In the first, the titanium rod was polished to ensure ideal wettability of its surface and to prevent carrying of any nuclei other than those already present in the liquid. It was then washed with dilute $\mathrm{HCl}$ and distilled water, dried and weighed accurately to four decimal figures. It was then joined to the velocity transformer and dipped in the melt as mentioned before. The oscillations produced from a HF generator fed to the magnetostrictive transducer, continued for $45 \mathrm{~min}$ at a driving amplitude of $4 \mu \mathrm{m}$.

The titanium rod was then taken away and left for $10 \mathrm{hr}$ in dilute $\mathrm{HCl}$ to ensure the removal of any attached lead, washed thoroughly with distilled water, dried and then weighed again to determine the weight loss. Experiments were carried out in this manner at $350,400,500,600,700$ and $800^{\circ} \mathrm{C}$ with two readings taken at each temperature.

In a separate experiment, a titanium rod was immersed directly $1 \mathrm{~cm}$ below the surface of the melt in a graphite crucible identical to that used before and left for $45 \mathrm{~min}$ at each temperature. No ultrasonic field was applied and the rod was weighed before and after each time, to determine the loss in its weight.

In the second series, spectrograms of cavitation noise spectrum as a function of temperature were recorded. The waveguide probe was placed in the cavitation zone where it received cavitation noise with practically uniform sensitivity. The signal was then fed to the input of the wave analyser where the output was recorded.

Cavitation threshold pressure of molten lead at different temperatures was obtained from a relation between subharmonic signals, and acoustic driving amplitude. The data were extracted from spectograms of cavitation noise at different acoustic amplitude and different temperatures (Abdel Fattah 1977).

\section{Results and discussion}

The experiment which was carried out when the titanium rod was immersed in molten lead and no ultrasonic field was applied, showed no change in weight before 
or after immersion. This indicates that titanium has suffered no erosion in molten lead at temperatures below $800^{\circ} \mathrm{C}$. Molten lead is known to be a chemically-negative environment that affect erosion process. From the lead-titanium phase diagram (Hansen and Anderko, 1958) titanium can be dissolved in lead only at temperatures above $725^{\circ} \mathrm{C}$. This indicates that the loss of weight in titanium rod, if any, is due mainly to the effect of ultrasonic waves in the molten lead.

The relationship between the loss of weight and temperature is shown in figure 1. It is clear from the figure that there is a slight increase in weight loss up to $600^{\circ} \mathrm{C}$, after which a sharp increase is noticed.

In figure 2 the relation between the weight loss and the cavitation threshold of molten lead at different temperatures is given. The increase in weight loss is more remarkable above $1.3 \mu \mathrm{m}$.

Figure 3 gives the cavitation noise spectrum in molten lead at different temperatures. The spectrograms shown indicate that the level of the cavitation noise spectrum, in general, increases slightly with temperature reaching a maximum at $700^{\circ} \mathrm{C}$.

Figure 4 shows microscopic photographs of the circular surface of titanium rods after exposure to ultrasonic waves at different temperatures. The pittings at the surface are more pronounced as the temperature increases.

The primary cause of cavitation erosion as reported by Plesset and Ellis (1961) is the mechanical stress developed from the motion of cavitating bubble (either

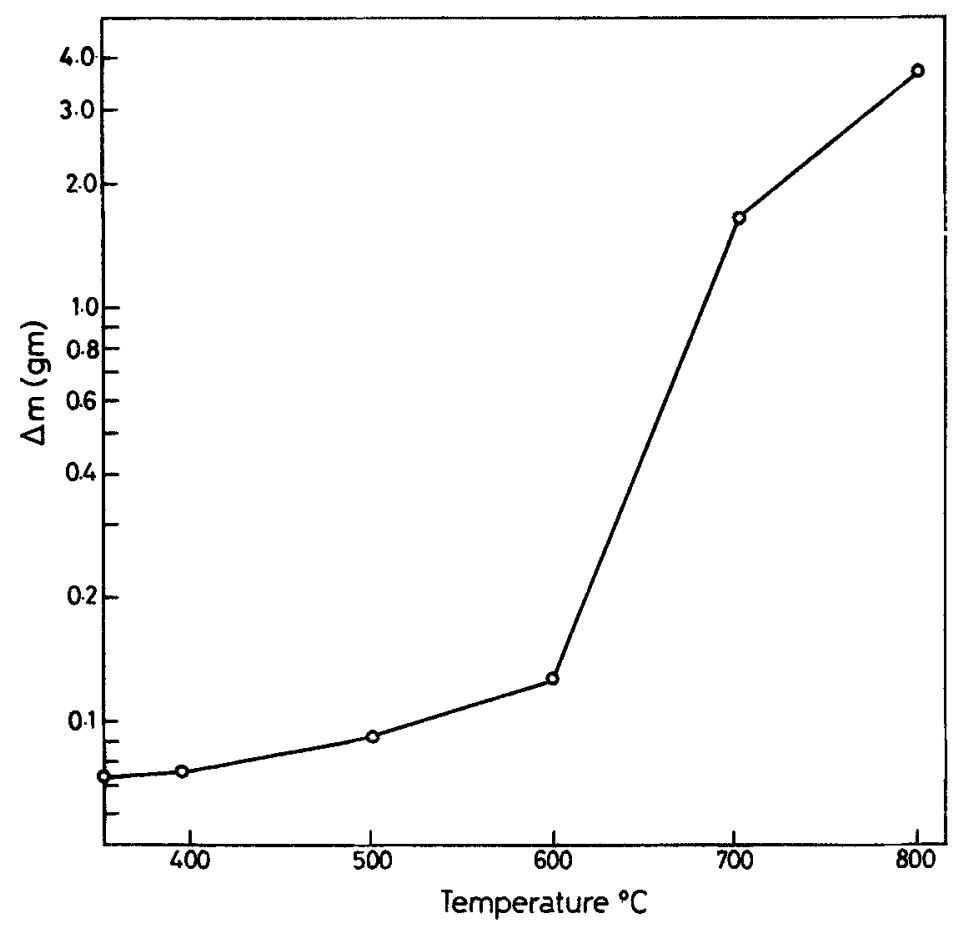

Figure 1. Cavitation erosion as a function of temperature. 


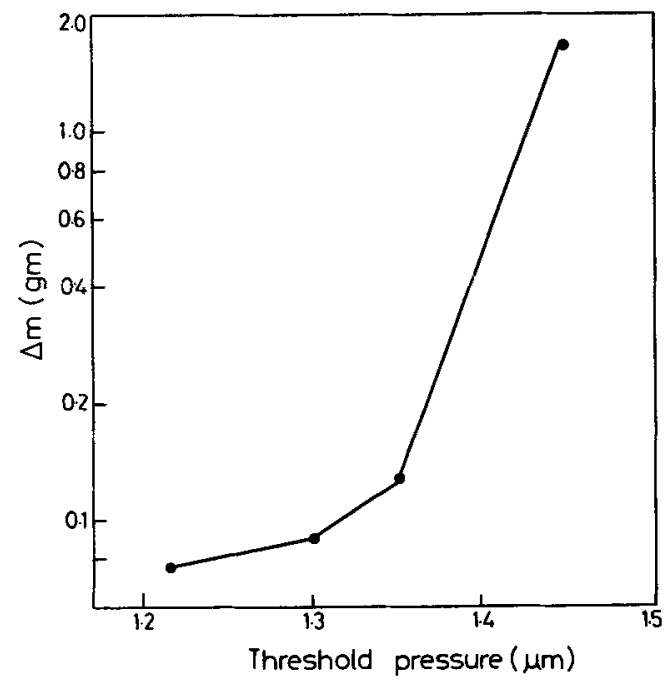

Figure 2. Cavitation erosion as a function of cavitation threshold pressure.

transient or stable cavities). However, Bebchuk (1957) reported that cavitation erosion can be caused by high speed jets arising from surface instabilities (shock waves).

The results reported by Rosenberg (1960), Bebchuk (1956) and Iernetti (1972) indicate that the cavitation erosion in different liquids exhibits a maximum at a definite temperature in the range between 0 and $60^{\circ} \mathrm{C}$. Our experiment was carried out at comparatively high temperatures and showed no maxima but gave gradual increase in weight loss with increasing temperature. An interesting observation is that the sharp increase in weight loss started at $600^{\circ} \mathrm{C}$, while it was observed from the phase diagram at $725^{\circ} \mathrm{C}$. This will be useful in metallurgy to produce titanium lead alloys.

The increase in weight loss with increasing temperature is due mainly to the fact that an increase in temperature will decrease the amount of gases present in the melt and a consequence of this is the increase in the shock wave energy. Also, as the ambient temperature increases, the thermal conductivity increases and hence there is an increase in the speed of contraction of a cavity leading to an increase of shock waves which is formed when a cavitational bubble collapses. Such an increase brings about an increase in cavitation activity. Although an increase in temperature increases the vapour pressure which plays a role in decreasing cavitation activity, there was a gradual increase in weight loss.

As shown in figure 2 cavitation erosion increases gradually with the threshold pressure which is confined to the stable type of cavitation. By increasing temperature this threshold increases although it is supposed to decrease, as mentioned by Sirotyuk (1966), in liquids at moderate temperatures. Considering the similarity between figures 1 and 2 we can attribute the increase in weight loss with increasing cavitation threshold to the dependance of erosion loss on temperature.

As shown in figure 1 the spectrograms of the cavitation noise consist of two types; the discrete harmonic components which are attributed to the stable type of cavitation 


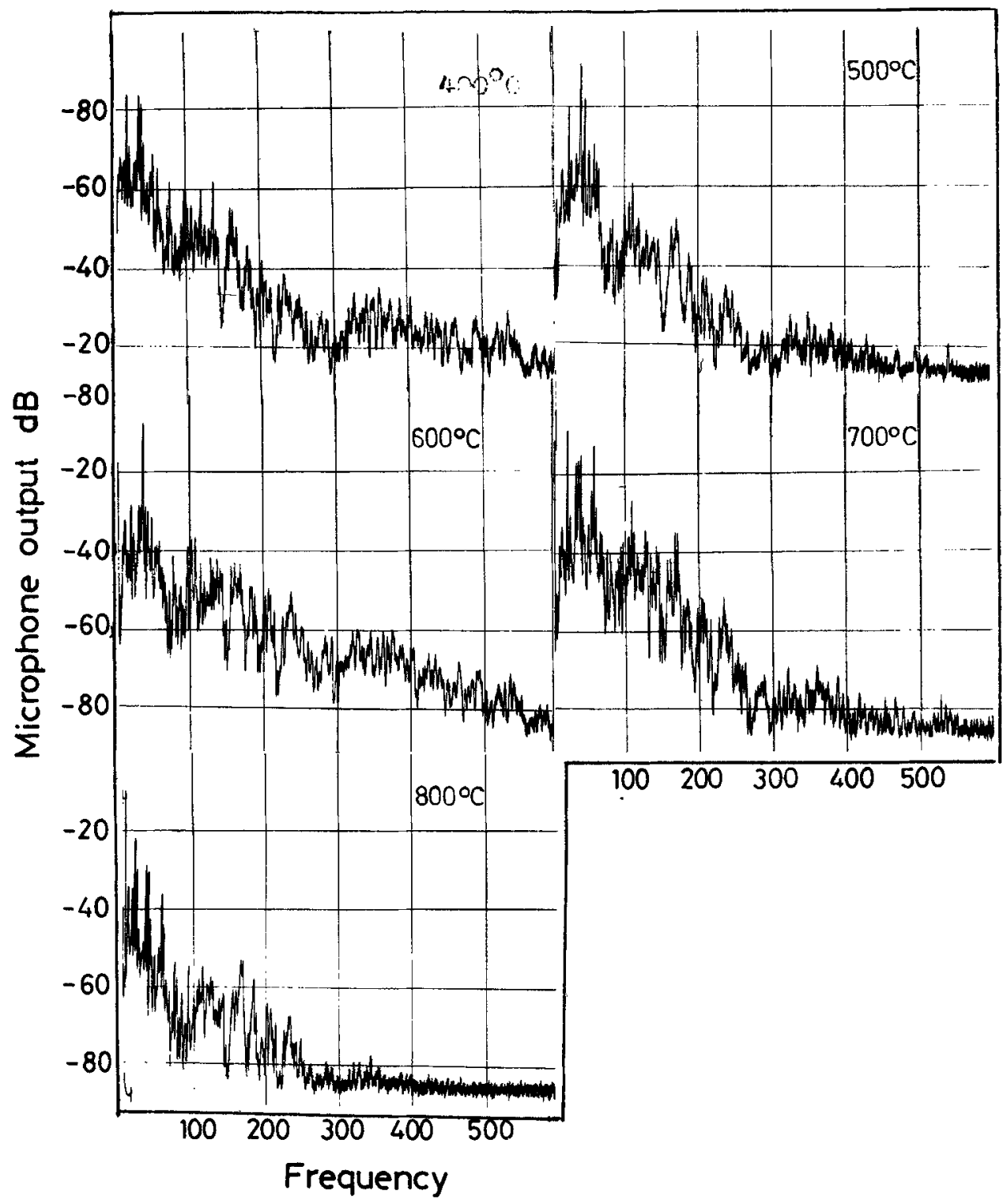

Figure 3. Cavitation noise spectrum in molten lead at different temperatures.

and the continuous part of spectrum attributed to transient type of cavitation. The level of both types in these spectrograms, in general, increases with increasing temperature. This increase is due to the effect of thermal conductivity and decreased gas content in the melt. Therefore, a quantitative measure of the noise level is needed to give the proper relationship. 

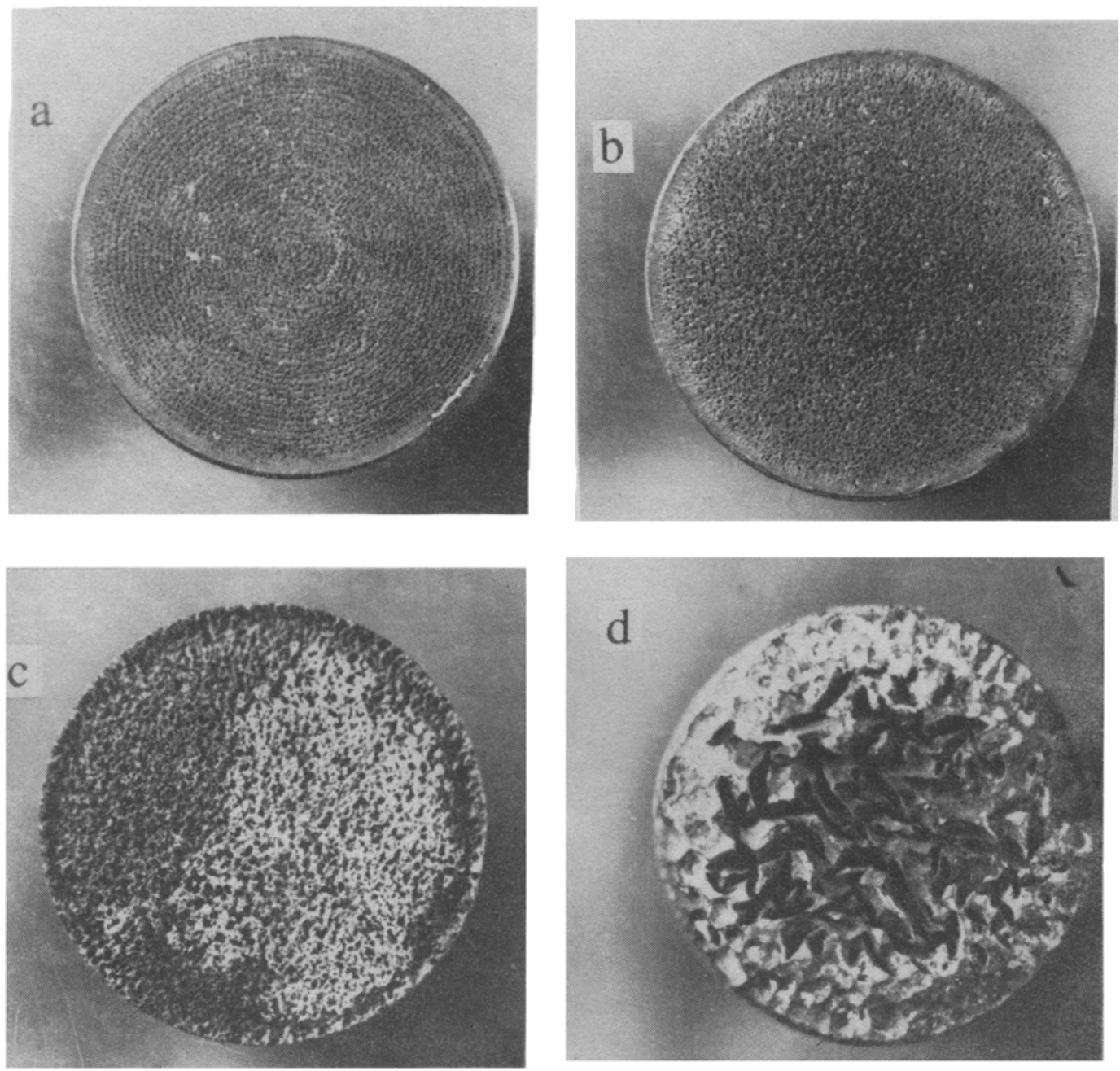

Figure 4. Microscopic photographs of titanium surfaces after exposure at (A) $500^{\circ} \mathrm{C}$ (B) $600^{\circ} \mathrm{C}(\mathbf{C}) 700^{\circ} \mathrm{C}$ and (D) $800^{\circ} \mathrm{C}$.

\section{References}

Abdel Fattah A M 1977 Cavitation spectra in molten metals by ultrasonics. Ph.D. Thesis, Cairo University Bebchuk A S 1956 Sov. Phys. Acoust. 295

Bebchuk A S 1957 Sov. Phys. Acoust. 3395

Bebchuck A S, Borisov Iv la and Rosenberg L. D 1958 Sov. Phys. Acoust. 4372

Esche R 1952 Akustiche Beihefte. 4208

Hansen M and Anderko K 1958 Constitution of binary alloys, (New York: MoGraw Hill) 1112

Ibisi M and Brown B 1967 Jour. Acoust. Soc. Am. 41568

Iernetti G 1972 Acoustica 26168

Plesset M S and Ellis A T 1961 Trans. Am. Soc. Mech. Engg. 771055

Rosenberg L D 1960 Ultrasonic News 416

Sirotvuk M G 1966 So' Phys Acrust. 1267 